\title{
Induced growth of BG-1 ovarian cancer cells by $17 \beta$-estradiol or various endocrine disrupting chemicals was reversed by resveratrol via downregulation of cell cycle progression
}

\author{
NAM-HEE KANG ${ }^{1 *}$, KYUNG-A HWANG $^{1 *}$, TAE-HEE KIM ${ }^{2}$, \\ SANG-HWAN HYUN ${ }^{1}$, EUI-BAE JEUNG ${ }^{1}$ and $\mathrm{KYUNG}^{-\mathrm{CHUL}} \mathrm{CHOI}^{1}$ \\ ${ }^{1}$ College of Veterinary Medicine, Chungbuk National University, Cheongju, Chungbuk; \\ ${ }^{2}$ Department of Obstetrics and Gynecology, College of Medicine, Soonchunhyang University, Bucheon, Republic of Korea
}

Received March 14, 2012; Accepted April 10, 2012

DOI: $10.3892 / \mathrm{mmr} .2012 .887$

\begin{abstract}
Resveratrol (trans-3,4',5-trihydroxystilbene; RES), a phytoestrogen, exists in grape skin and red wine. Endocrine disrupting chemicals (EDCs) appear to promote the development and progression of estrogen-dependent cancers. In this study, we evaluated the inhibitory effect of RES on the cell proliferation induced by various EDCs in BG-1 ovarian cancer cells. Various EDCs, such as bisphenol A (BPA), nonylphenol (NP), octylphenol (OP), methoxychlor (MXC) and hexabromocyclododecane (HBCD), were employed in this study. In the in vitro experiments, treatment of BG-1 cells with E2, BPA, NP, $\mathrm{OP}, \mathrm{MXC}$ or HBCD resulted in an increase of cell growth. By contrast, increased cell viability induced by these EDCs was reversed when co-cultured with RES. In addition, we examined the regulation of cell cycle-related genes by RT-PCR and western blot analysis. Treatment with each EDC was found to decrease only the gene expression of $p 21$ and increase the expression of cell cycle-dependent kinase 2 (CDK2). However, co-treatment with RES and each EDC resulted in an increased gene expression of p21 and a decreased expression of CDK2. Cyclin D1 was increased by downregulating $p 21$ only when treated with each EDC in the absence of RES, while co-treatment with RES and each EDC decreased the gene expression of cyclin DI by upregulating $p 21$. Taken together, RES appears to be an inhibitor of cyclin D1 and CDK2 and is responsible for the cell cycle arrest at the $\mathrm{G}_{1}$ phase. In addition, when co-treated with each EDC, RES increased the expression of $p 21$ and resulted
\end{abstract}

Correspondence to: Dr Kyung-Chul Choi, Laboratory of Veterinary Biochemistry and Immunology, College of Veterinary Medicine, Chungbuk National University, Cheongju, Chungbuk 361-763, Republic of Korea

E-mail:kchoi@cbu.ac.kr

${ }^{*}$ Contributed equally

Key words: resveratrol, cell proliferation, cell cycle arrest, p21, cyclin D1, cyclin-dependent kinase 2 in the growth inhibition of BG-1 ovarian cancer cells. Taken together, these results indicate the antiproliferative effect of RES, a dietary phytoestrogen, on estrogen-dependent ovarian cancer cells activated by EDCs.

\section{Introduction}

Endocrine disrupting chemicals (EDCs) are the environmental chemicals that interfere with endocrine systems by adversely affecting hormone balance or disrupting normal function in the organs that hormones regulate or modulate (1). EDCs, such as bisphenol A (BPA), 4-nonylphenol (NP), 4-tert-octylphenol (OP), methoxychlor (MXC) and hexabromocyclododecane (HBCD), are released from industrial products, such as plastics, pesticides, detergents and other chemosynthetic products (2). EDCs lead to serious detrimental effects on the reproductive and developmental processes of human, animals and plants (3). They possess the potential to increase human health risks and affect the immune system and the development of vital organs. In addition, it has been reported that EDCs may increase the risk of cancer incidence (2). For example, the cell growth of MCF-7 breast cancer cells was increased by MXC and BPA in vitro (4). The cell proliferation of BG-1 ovarian adenocarcinoma cells was promoted by di-n-butyl phthalate (DBP) and HBCD through upregulation of the expression of cyclin-dependent kinase 4 (CDK4). These results suggest that DBP and HBCD have sufficient potency to disrupt the endocrine system and to stimulate cell growth in estrogen receptor (ER)-positive BG-1 cancer cells in vitro through the upregulation of genes that are associated with cell cycle progression (5). Consequently, these EDCs may be carcinogenic. Therefore, therapeutic tools for reducing the risk of carcinogenicity resulting from the exposure of EDCs are required.

Ovarian carcinoma is one of the most frequent gynaecologic cancers, affecting more than 200,000 female individuals annually worldwide. Findings of a recent study showed that $p 21$ effectively suppresses cancer growth by enhancing apoptosis and DNA damage response in ovarian cancer (7). Therefore, an investigation was conducted to identify an anticancer agent in natural food that enhances the expression of $p 21$. Additionally, an anticancer therapy was examined for the cancers promoted 
by EDCs using resveratrol (RES) in the BG-1 ovarian adenocarcinoma cell line, a well-known estrogen-dependent cell line expressing ERs, including $\operatorname{ER} \alpha$ and $\operatorname{ER} \beta(3,6)$.

RES, a natural polyphenolic compound found in a variety of food sources, such as grapes, peanuts and red wine, is known for its anti-oxidant and anti-inflammatory effects, cardiovascular protection and increases longevity. RES has a strong chemopreventive effect (8) against the development of cancers, such as skin, breast, prostate, lung, pancreatic and ovarian cancer, by inhibiting cancer cell growth (9-13). RES also promotes the phosphorylation of p53 in a dose- and timedependent manner in human breast cancer cells. Thus, as p53 is activated, it causes either cell cycle arrest or apoptosis. In addition, the expression of $p 21$ is upregulated by the increased expression of $p 53$, leading to cancer cell death. In this study, the inhibitory effect of RES on cell proliferation of the BG-1 ovarian adenocarcinoma cell line, which was stimulated by the exposure of EDCs, was also evaluated. EDCs, such as BPA, NP and OP, appeared to upregulate cyclin DI and cyclindependent kinase 2 (CDK2), which is responsible for cell cycle progression. By contrast, RES increased the expression of $p 21$, the CDK inhibitor, leading to the suppression of cancer cell growth.

\section{Materials and methods}

Reagents and chemicals. 17 $\beta$-estradiol (E2), BPA, NP, OP, MXC, HBCD and RES were purchased from Sigma-Aldrich Corp. (St. Louis, MO, USA). Chemicals were dissolved in $100 \%$ dimethyl sulfoxide (DMSO; Junsei Chemical Co., Tokyo, Japan) and stored as stock solutions at $4^{\circ} \mathrm{C}$.

Cell culture. The BG-1 ovarian adenocarcinoma cell line was obtained from Dr K.S. Korach (National Institute of Environmental Health Sciences, Research Triangle Park, NC, USA). The cells were cultured in Dulbecco's modified Eagle's medium (DMEM; Hyclone Laboratories Inc., Logan, UT, USA) supplemented with $10 \%$ heat-inactivated fetal bovine serum (FBS; Hyclone Laboratories Inc.), $1 \%$ penicillin G, streptomycin (Cellgro Mediatech, Inc., Manassas, VA, USA) and 1\% antifungal HEPES (Invitrogen Life Technologies, Carlsbad, $\mathrm{CA}, \mathrm{USA}$ ) at $37^{\circ} \mathrm{C}$ in a humidified atmosphere of $5 \% \mathrm{CO}_{2}-95 \%$ air. To prevent the effects of the estrogenic components of DMEM and FBS, phenol red-free DMEM supplemented with $5 \%$ charcoal-dextran-treated FBS (CD-FBS) was used to rule out the estrogenicity of EDCs in BG-1 cells. Cells were detached with $0.05 \%$ trypsin/ $0.02 \%$ EDTA in $\mathrm{Mg}^{2+} / \mathrm{Ca}^{2+}$-free Hank's balanced salt solution (HBSS; PAA Laboratories Ltd., Linz, Austria ).

Cell proliferation assay. To examine the anticancer effect by RES, a 3-(4,5-dimethylthiazol-2-yl)-2,5-diphenyltetrazolium bromide (MTT) assay was performed. BG-1 cells were plated at 4,000 cells/well in 96-well plates in $100 \mu \mathrm{l}$ of phenol red-free DMEM with 5\% CD-FBS medium. After a 48-h incubation, the cells were washed and treated with RES at concentrations of $0,10,25,50,75$ and $100 \mu \mathrm{M}$ in phenol red-free DMEM with $0.1 \%$ of DMSO (Junsei Chemical Co.), respectively, for 5 days. Cell viability was assessed using an MTT assay. MTT (10 $\mu \mathrm{l}$; Sigma-Aldrich Corp.) solution (5 mg/ml) was added to each well and the plates were incubated for $4 \mathrm{~h}$ at $37^{\circ} \mathrm{C}$. Supernatants were removed and $100 \mu \mathrm{l}$ of $99.0 \%$ DMSO (Junsei Chemical Co.) was added to each well to dissolve the resultant formazan crystals. Optical densities were measured at a wavelength of $540 \mathrm{~nm}$ using an ELISA-Reader (VERSA man; Molecular Devices, Sunnyvale, CA, USA).

To evaluate the cell proliferative effect on BG-1 cells, the various EDCs were treated. BG-1 cells were plated in 96-well plates of 4,000 cells/well in $100 \mu \mathrm{l}$ of phenol red-free DMEM with $5 \%$ CD-FBS medium. After a 48-h incubation, the cells were washed and treated with DMSO, E2, BPA, NP, OP, $\mathrm{HBCD}, \mathrm{MXC}$ and RES at concentrations of $0.1 \%, 10^{-9}, 10^{-5}$, $10^{-5}, 10^{-5}, 2 \times 10^{-7}, 10^{-6}$ and $10^{-4} \mathrm{M}$, respectively. Co-treatment of RES with each EDC was conducted at the time for 5 days and cell viability was assessed using MTT assay.

Total RNA extraction and cDNA synthesis. BG-1 cells were cultured at $3 \times 10^{5}$ cells/well of 6-well plates and DMSO, E2, BPA, NP, RES and the combination of RES with each EDC were treated. Total RNA was extracted at various timepoints $(0,6$ and $24 \mathrm{~h})$ using TRIzol reagents (Invitrogen Life Technologies) according to the manufacturer's instructions. The concentration of total RNAs was measured by a spectrophotometer (Optizen, Mecasys, Dea-jeon, Korea) at 260/280 $\mathrm{nm}$. Total RNA (1 $\mu \mathrm{g})$ was dissolved in dietyl pyrocarbonated-deionized water for cDNA synthesis (14).

Semi-quantitative reverse transcription $(R T)-P C R$. cDNA was synthesized from total RNAs by RT-PCR. Reaction mixture comprised murine leukemia virus reverse transcriptase (M-MLV RT; iNtRON Biotechnology, Sungnam, Kyeonggido, Korea), 200 pM nonamer random primer, dNTPs, RNase inhibitor and RT buffer (all from iNtRON Biotechnology). cDNA synthesis was performed at $37^{\circ} \mathrm{C}$ for $1 \mathrm{~h}$ and at $95^{\circ} \mathrm{C}$ for 5 min. cDNA of $p 21, C D K 2$, cyclin D1 and GAPDH was amplified using each forward and reverse primer. Taq polymerase, PCR buffer, dNTP mixture and each cDNA template were conducted via PCR process, as described previously $(5,14,15)$ (Table I). The RT-PCR products were run on a $1.5 \%$ agarose gel and gene bands were compared to 100-bp ladders (iNtRON Biotechnology). The gels were scanned and the density of the bands on the gel was quantified using Gel Doc 2000 (Bio-Rad Laboratories, Inc., Hercules, CA, USA) $(15,16)$.

Western blot analysis. Western blotting was performed to identify the protein expression of $p 21$ and cyclin D1 in BG-1 cells. BG-1 cells were cultured at $1 \times 10^{6}$ cells/100-mm dish and $0.1 \%$ DMSO, E2, BPA, RES and the combination of RES plus E2 or BPA were treated. Cells were suspended in $100 \mu \mathrm{l}$ of $1 \mathrm{X}$ RIPA lysis buffer. The protein concentrations were determined using a bicinchoninic acid method. Total protein $(50 \mu \mathrm{g})$ was run on $10 \%$ sodium dodecyl sulfate-polyacrylamide gel electrophoresis (SDS-PAGE) and transferred to a polyvinylidene difluoride (PVDF) membrane (Bio-Rad Laboratories, Inc.). PVDF membranes were blocked with $5 \%$ bovine serum albumin (Sigma-Aldrich Corp.) for $2 \mathrm{~h}$ at room temperature. The membranes were incubated with mouse monoclonal anti-p21 antibody (1:4,000; Cell Signaling Technology, Inc., Danvers, MA, USA), mouse monoclonal anti-cyclin D1 antibody (1:2,000; Abcam, Hanam-city, Gyeonggi-do, Korea) 
Table I. Primer sequences and sizes for the semi-quantitative reverse transcription-PCR.

Target gene

p21

$C D K 2$

Cyclin DI

GAPDH
Sequences

Product size (bp)

Sense: 5'-AGGCACCGAGGCACTCAGAG-3'

370

Antisense: 5'-TGACAGGTCCACATGGTCTTCC-3'

Sense: 5'-CATTCCTCTTCCCCTCATCA-3'

Antisense: 5'-TGGGGAAACTTGGCTTGTAA-3'

Sense: 5'-TCTAAGATGAAGGAGACCATC-3'

354

Antisense: 5'-GCGGTAGTAGGACAGGAAGTTGTT-3'

Sense: 5'-ATGTTCGTCATGGGTGTGAACCA-3'

351 and mouse monoclonal anti-GAPDH antibody (1:1,000; Santa Cruz Biotechnology, Santa Cruz, CA, USA) for $2 \mathrm{~h}$ at room temperature. The membranes were subsequently probed with anti-mouse IgG-HRP conjugate secondary antibody (1:3,000; Santa Cruz Biotechnology). Target proteins were detected with a West-Q Chemiluminescent Substrate kit Plus (GenDEPOT, Barker, TX, USA).

Statistical analysis. Data were analyzed with GraphPad Prism software (San Diego, CA, USA). The in vitro data were shown as the means \pm SD. Statistical analysis was performed using the one-way ANOVA followed by Dunnett's multiple comparison test and T-test. $\mathrm{P}<0.05$ was considered statistically significant $(17,18)$.

\section{Results}

Effects of RES, E2, EDCs or their combinations on the cell proliferation of $B G-1$ cells. To evaluate the effect on cell proliferation by RES, BG-1 cells were cultured with a vehicle (DMSO $0.1 \%$ ) or RES (10-100 $\mu \mathrm{M})$ for 5 days. RES effectively reduced the cell viability of BG-1 cells in a dose-dependent manner (Fig. 1A). When treated with $100 \mu \mathrm{M}$ of RES, the viability of BG-1 cells was decreased by $64 \%$ compared to that of the vehicle. The effects of E2, several EDCs and their combinations with RES were examined on the viability of BG-1 cells. E2 and EDCs, such as BPA, NP, OP, HBCD and $\mathrm{MXC}$, consequently increased the cell growth of BG-1 cells by $80,69,63,47,23$ and $28 \%$, respectively, compared to the vehicle (Fig. 1B). By contrast, by adding RES to each treatment, the proliferative effect of E2 or each EDC was significantly suppressed by RES. RES reversed the increased cell growth by E2, BPA, NP, OP, HBCD and MXC by 54, 63, 43, 62 and 63\%, respectively (Fig. 1B).

Effects of E2, BPA, NP, RES or their combinations on the expression of cell cycle-related genes. To evaluate the effect of RES on the expression levels of genes associated with the cell cycle, we treated BG-1 cells with E2, BPA, NP, RES or their combinations, and performed semi-quantitative RT-PCR for mRNA expression isolated from the cells at 0,6 and $24 \mathrm{~h}$ in a time-dependent manner. The gene expression of $p 21$ was significantly decreased by E2 at 6 and 24 h compared to the vehicle. By contrast, $p 21$ expression was significantly increased by the treatment of RES, E2 plus RES, or BPA
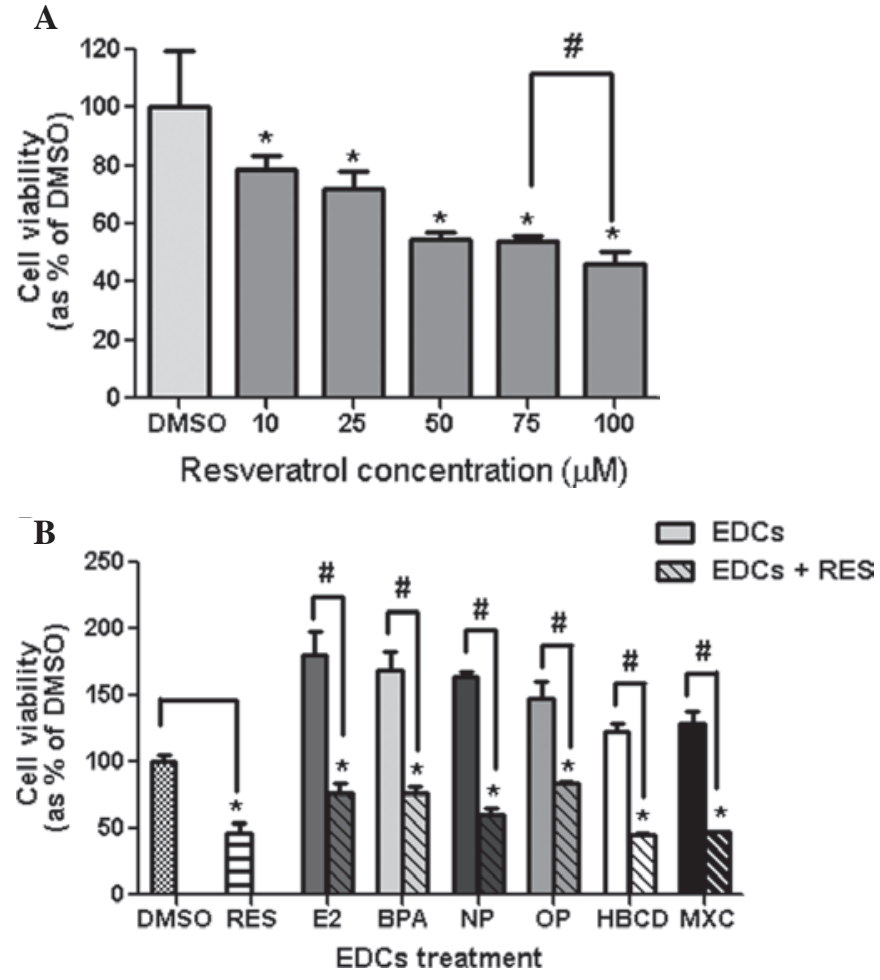

Figure 1. Cell viability on BG-1 cells by MTT assay. BG-1 cells were plated at 4,000 cells/well in $100 \mu 1$ of phenol red-free DMEM with 5\% CD-FBS medium. (A) The cells were washed and treated with RES at concentrations of $0,10,25,50,75$ and $100 \mu \mathrm{M}$ in phenol red-free DMEM with $0.1 \%$ of DMSO for 5 days. Values are the means \pm SD. *Significant elevation compared to vehicle (0.1\% DMSO), $\mathrm{P}<0.05$ (Dunnett's multiple comparison test). "Significant elevation compared to $75 \mu \mathrm{M}$ or RES, $\mathrm{P}<0.05$ (t-tests). (B) Various EDCs were treated. After incubation for $48 \mathrm{~h}$, the cells were washed and treated with DMSO, E2, BPA, NP, OP, HBCD, MXC and RES at concentrations of $0.1 \%, 10^{-9}, 10^{-5}, 10^{-5}, 10^{-5}, 2 \times 10^{-7}, 10^{-6}$ and $10^{-4} \mathrm{M}$, respectively. Co-treatment of RES with each EDC was conducted at the time for 5 days and cell viability was assessed by MTT assay. Values are the means \pm SD. *Significant elevation compared to vehicle (0.1\% DMSO), $\mathrm{P}<0.05$ (Dunnett's multiple comparison test). "Significant elevation compared to E2 or each EDC treatment group, $\mathrm{P}<0.05$ (t-tests).

plus RES at $24 \mathrm{~h}$ (Fig. 2A). The gene expression of cyclin Dl was significantly increased by E2 and BPA at 6 and $24 \mathrm{~h}$, but markedly decreased by RES, E2 plus RES, or BPA plus RES treatment (Fig. 2B). The gene expression of $C D K 2$ was considerably increased by E2 and BPA at 6 and $24 \mathrm{~h}$. However, it was significantly decreased by the treatment of RES, E2 plus RES, or BPA plus RES treatment (Fig. 2C). In addition, the gene 

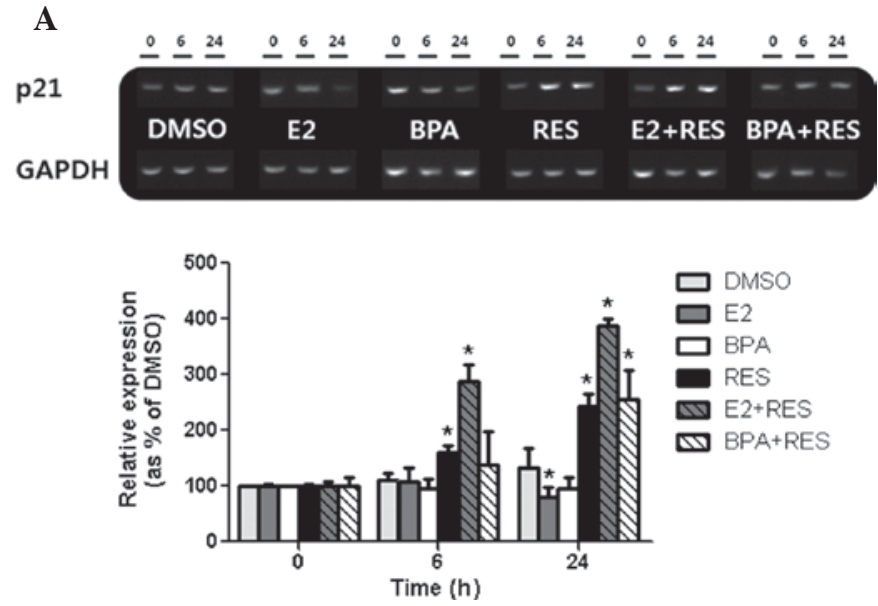

B
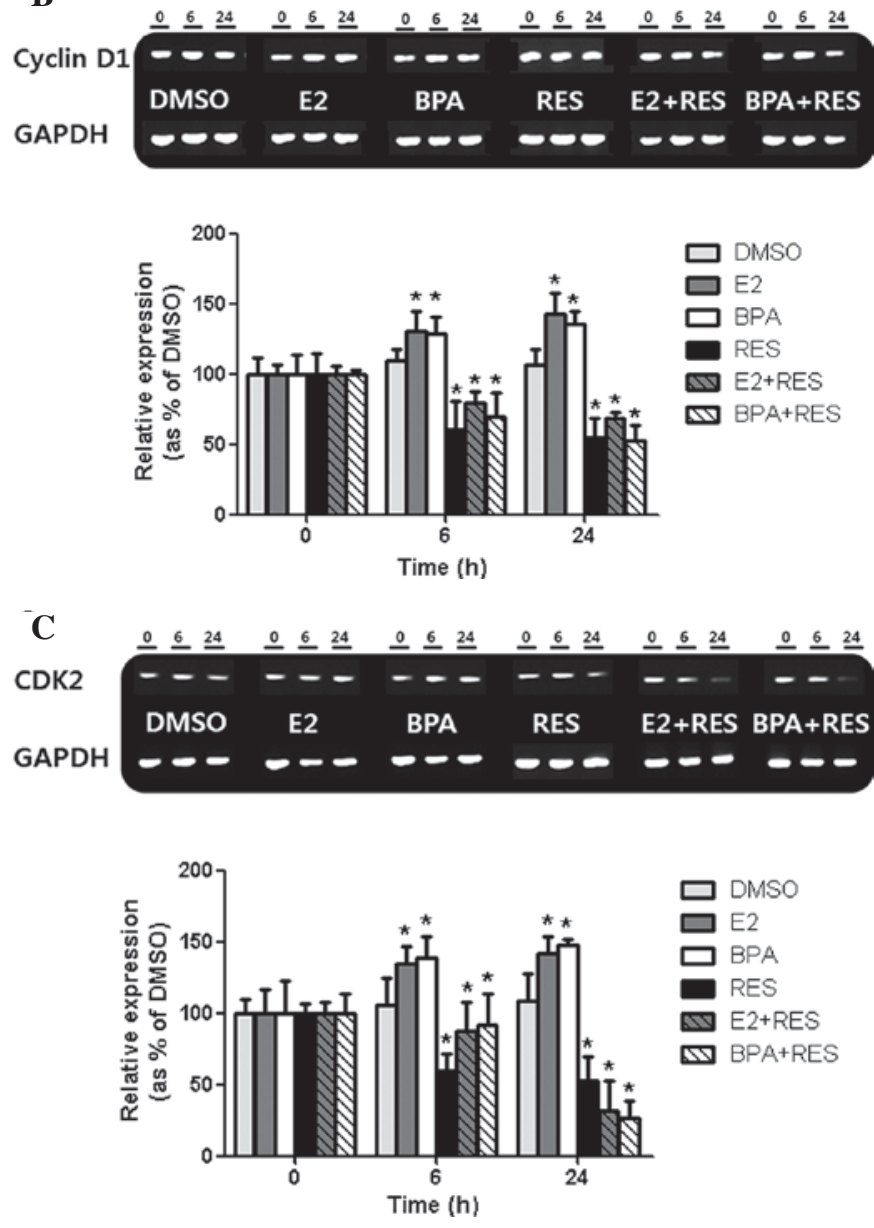

Figure 2. Alteration of cell cycle-related genes following treatment with RES or BPA. BG-1 cells were cultured at $3 \times 10^{5}$ cells/well of 6 -well plates and $0.1 \%$ DMSO, E2, BPA, RES and the combination of RES with E2 or BPA were treated. Total RNA was extracted at various time-points $(0,6$ and $24 \mathrm{~h})$ using TRIzol reagents. cDNA was synthesized from total RNAs by RT-PCR. cDNAs of (A) $p 21$, (B) cyclin D1, (C) CDK2 and GAPDH were amplified. The RT-PCR products were run on a $1.5 \%$ agarose gel. The gels were scanned and the density of the bands on the gel was quantified using Gel Doc. Values are the means \pm SD. ${ }^{*} \mathrm{P}<0.05$ (Dunnett's multiple comparison test).

expression of $p 21$ was significantly decreased by E2 and NP at $24 \mathrm{~h}$, while its expression levels were significantly increased at 6 and $24 \mathrm{~h}$ in the presence of RES and its combinations (Fig. 3A). The cyclin D1 expression levels of E2 and NP were increased at 6 and $24 \mathrm{~h}$, but markedly decreased by RES, E2
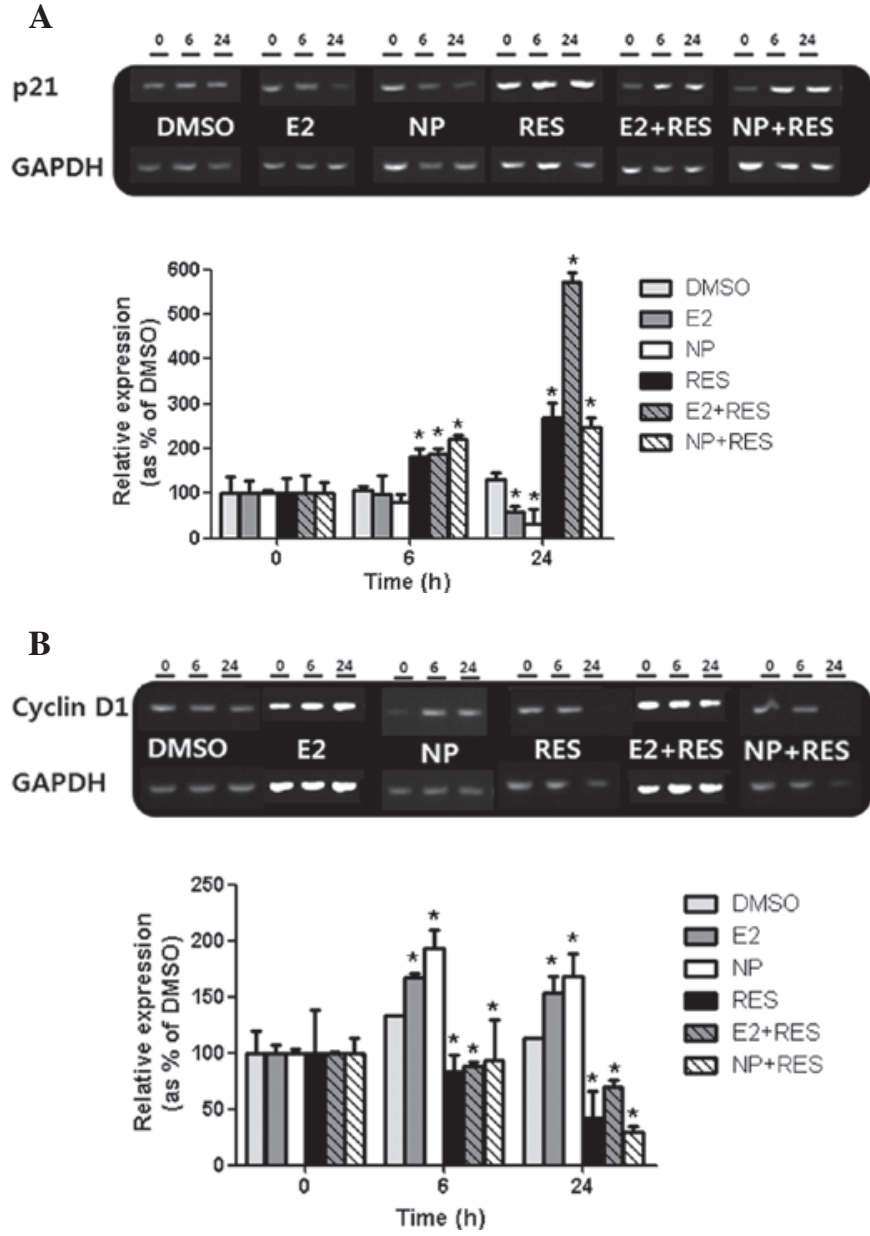

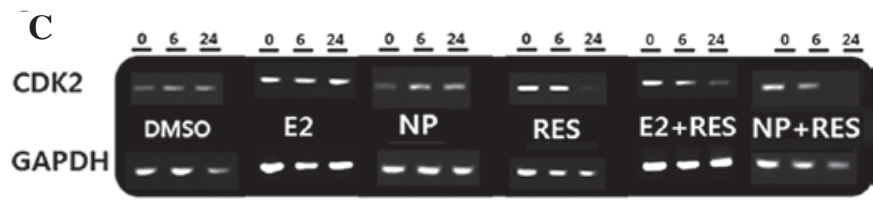

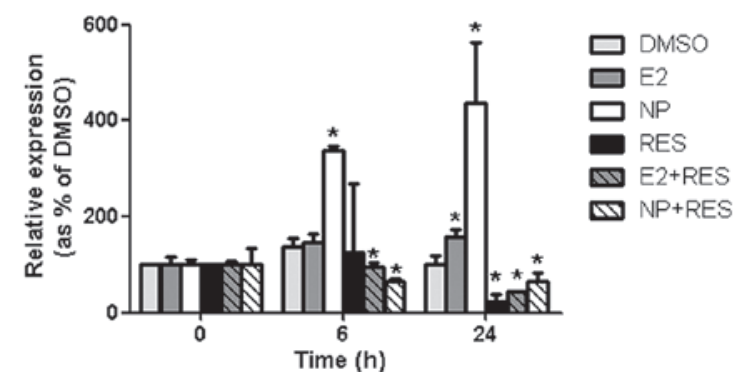

Figure 3. Alteration of cell cycle-related genes following treatment with RES or NP. BG-1 cells were cultured at $3 \times 10^{5}$ cells/well of 6-well plates and treated with $0.1 \%$ DMSO, E2, NP, RES and the combination of RES plus E2 or NP. The gene expression was detected as described in Materials and methods. The expression of (A) $p 21$, (B) cyclin D1 and (C) CDK2 was indicated. Values are the means $\pm \mathrm{SD}$. ${ }^{*} \mathrm{P}<0.05$ (Dunnett's multiple comparison test).

plus RES, or NP plus RES (Fig. 3B). The gene expression of $C D K 2$ was considerably increased by E2 and NP at 6 and $24 \mathrm{~h}$ compared to the vehicle, but significantly decreased by RES and its combinations with E2 or NP (Fig. 3C).

Effects of E2, BPA, RES or their combinations on the protein expression of cell cycle-related genes. To investigate whether 

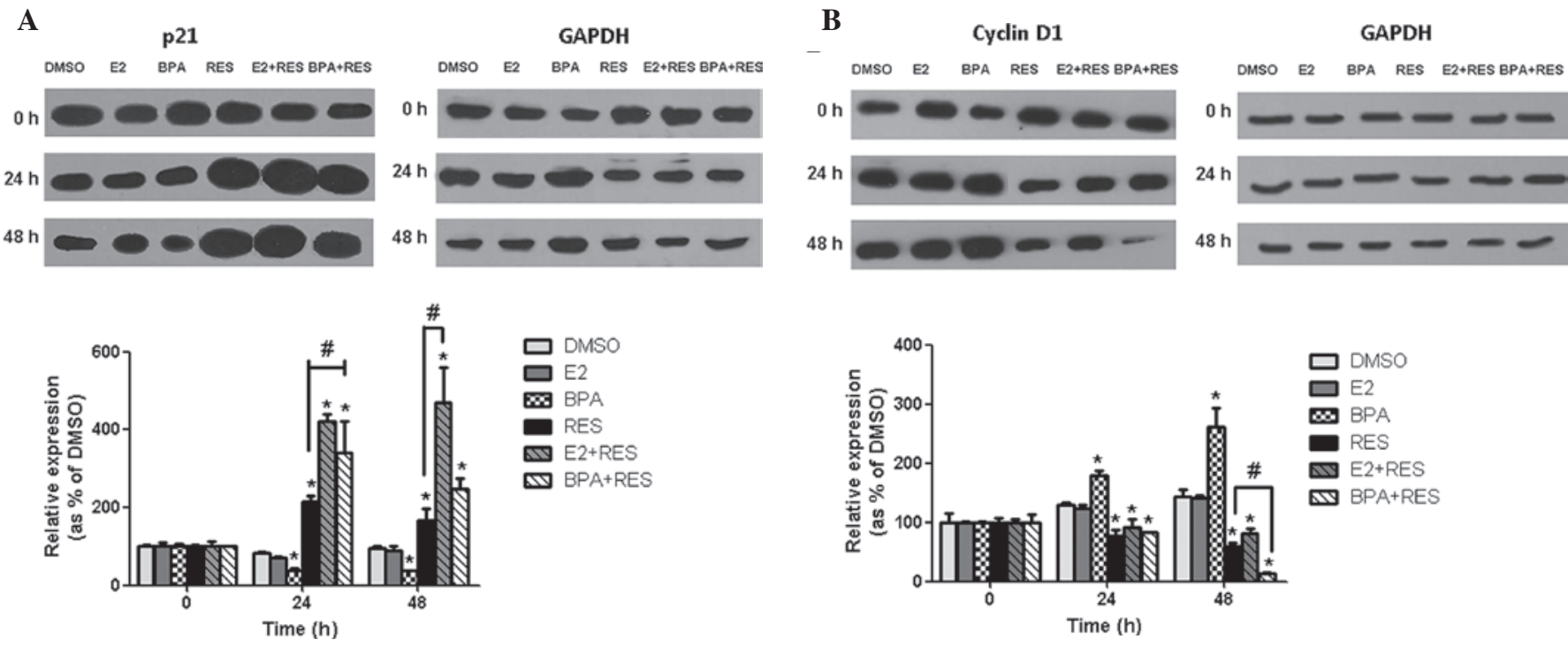

Figure 4. Alteration of cell cycle-related proteins following treatment with RES and diverse EDCs. BG-1 cells were cultured at 1x10 cells/100-mm dish, and treated with 0.1\% DMSO, E2, BPA, RES and the combination of RES plus E2 or BPA. Western blot analysis was performed to identify the protein expression of (A) p21 and (B) cyclin D1 in BG-1 cells. Values are the means \pm SD. "Significant elevation compared to the vehicle ( $0.1 \%$ DMSO), P $<0.05$ (Dunnett's multiple comparison test). " Significant elevation compared to RES, $\mathrm{P}<0.05$ (t-tests).

E2, BPA, RES or their combinations alter the protein expression of cell cycle-related genes in the BG-1 human ovarian cancer cell line, western blot analysis was performed for the protein samples isolated from the cells at 0,24 and $48 \mathrm{~h}$ in a time-dependent manner. The protein levels of $p 21$ and cyclin D1 were measured following treatment with E2, BPA, RES, E2 plus RES or BPA plus RES in BG-1 cells. Findings showed the protein levels of $p 21$ to be significantly decreased by E2 and BPA compared to the vehicle at 24 and $48 \mathrm{~h}$, whereas the levels were markedly increased by RES, E2 plus RES, or BPA plus RES compared to the vehicle (Fig. 4). When treated with E2 plus RES, or BPA plus RES, the $p 21$ protein exhibited a stronger expression compared to RES only at $24 \mathrm{~h}$.

\section{Discussion}

EDCs are chemical compounds that are widely distributed in the environment, diet or various chemosynthetic products. Various EDCs exhibit estrogenic activity and interfere with normal ER-dependent signaling pathways mediated by two ERs, such as ER $\alpha$ and ER $\beta$. A number of EDCs directly impact ER signaling by competing with endogenous estrogen for ER binding sites (19). In general, EDCs tend to increase the cell proliferation of ER-expressing cells, such as ovarian cancer cells, by exerting estrogenic activity in vitro and in vivo.

Ovarian cancer is the leading cause of death from gynecologic malignancies $(3,20)$. The median survival rate of ovarian cancer patients in advanced stage is 5 years and remains low, although anticancer therapies are currently being examined (21). Results of the present study demonstrated that RES effectively inhibited the cell proliferation increased by EDCs on BG-1 cells expressing both ERs by inducing cell cycle arrest. From previous studies, RES is known for exerting antitumorigenic effects by suppressing cell proliferation and inducing apoptosis in various cancer cells, such as colon and pancreatic cancer cells $(22,23)$.
Finding of this study showed that BG-1 cell growth was promoted by E2 or various EDCs, including BPA, NP, OP, MXC and HBCD. These EDCs may induce BG-1 cell proliferation by affecting estrogen signaling via ERs. To evaluate the inhibitory effect of RES on the growth of BG-1 cells, RES was treated in a single treatment or in combination with E2 or other EDCs. Consequently, the cell viability and cell growth of BG-1 cells previously promoted by these EDCs were significantly suppressed by RES, which was considered to be associated with the regulation of cell cycle-related genes. Regulation of the cell cycle is closely involved in tumor cell proliferation, differentiation and apoptosis. Cell cycle progression is coordinately regulated by a number of protein factors that mainly include cyclins, CDKs and cyclin-dependent kinase inhibitors $(C K I s)$. The regulation of $C D K$ activation is crucial during cell cycle changes and is affected by cyclins (24). Cyclin DI is an important protein for cell cycle progression and is known to function as a regulatory subunit of $C D K 4$ or $C D K 6$, whose activity is required for the $G_{0} / G_{1}-S$ phase transition (23). The activities of $C D K s$ are also inhibited by $p 21, p 27$ and $p 57$, which have a nuclear localization signal at the C-terminus (24). This group of proteins exerts its inhibitory activities by interacting with a variety of cyclin-CDK complexes, thereby inducing S-phase arrest. Thus, the increased expression of p21 blocks the expression of the cell cycle progression factor and leads to increased $\mathrm{G}_{0}-\mathrm{G}_{1}$ cell cycle arrest. In addition, the increased $p 21$ protein may contribute to enhanced cell apoptosis and genomic instability.

In this study, $p 21$, cyclin $D 1$ and $C D K 2$ were observed as the main factors affecting cell viability or cell proliferation. The gene expression of cyclin D1 and CDK2 was considerably decreased by RES in a time-dependent manner. However, the gene expression of $p 21$ was significantly increased by RES, which is considered to inhibit the expression of cell cycle progression factors, i.e., cyclin $D 1$ and $C D K 2$, and induce cell apoptosis through $\mathrm{G}_{0}-\mathrm{G}_{1}$ cell cycle arrest. The expression 
patterns of $p 21$ and cyclin D1 proteins by E2, EDCs and RES were detected concomitantly with their gene expression, i.e., the protein levels of $p 21$ were significantly increased and cyclin Dl was markedly decreased by RES.

Our findings demonstrated that whereas E2 and several EDCs promoted the cell proliferation of ovarian adenocarcinoma BG-1 cells expressing ERs, RES effectively inhibited cell proliferation. As shown in this study, the cell proliferation of ovarian cancer cells by EDCs suggests that certain EDCs possess carcinogenic or cancer-promoting risks. However, RES showed anticancer activity on BG-1 ovarian cancer by reversing the cell proliferative effects induced by EDCs. Taken together, these results indicate that RES suppresses ovarian cancer cell growth by inducing cell cycle arrest. Although the cell cycle progression factors, i.e., cyclin $D 1$ and $C D K 2$, were inhibited, $p 21$ protein, the cell cycle arrest factor that blocks a variety of cyclin-CDK complexes, was upregulated by RES. Therefore, we suggest the therapeutic effect and underlying mechanism of RES as a chemotherapeutic agent against estrogen responsive cancers whose cell growth may be induced by EDCs.

\section{Acknowledgements}

This study was supported by a National Research Foundation of Korea (NRF) grant (no. 2011-0015385), funded by the Ministry of Education, Science and Technology (MEST) of the Korea Government.

\section{References}

1. Choi KC and Jeung EB: The biomarker and endocrine disruptors in mammals. J Reprod Dev 49: 337-345, 2003.

2. Park SH, Kim KY, An BS, Choi JH, Jeung EB, Leung PC and Choi KC: Cell growth of ovarian cancer cells is stimulated by xenoestrogens through an estrogen-dependent pathway, but their stimulation of cell growth appears not to be involved in the activation of the mitogen-activated protein kinases ERK-1 and p38. J Reprod Dev 55: 23-29, 2009.

3. Hwang KA, Park SH, Yi BR and Choi KC: Gene alterations of ovarian cancer cells expressing estrogen receptors by estrogen and bisphenol a using microarray analysis. Lab Anim Res 27: 99-107, 2011.

4. Lee HR, Hwang KA, Park MA, Yi BR, Jeung EB and Choi KC: Treatment with bisphenol $\mathrm{A}$ and methoxychlor results in the growth of cycle-related genes, cyclin D1 and p21, via an estrogen receptor-dependent signaling pathway. Int J Mol Med 29: 883-890, 2012.

5. Park MA, Hwang KA, Lee HR, Yi BR, Jeung EB and Choi KC: Cell growth of BG-1 ovarian cancer cells is promoted by di-nbutyl phthalate and hexabromocyclododecane via upregulation of the cyclin D and cyclin-dependent kinase-4 genes. Mol Med Report 5: 761-766, 2011.

6. Geisinger KR, Kute TE, Pettenati MJ, Welander CE, Dennard Y, Collins LA and Berens ME: Characterization of a human ovarian carcinoma cell line with estrogen and progesterone receptors. Cancer 63: 280-288, 1989.

7. Yang G, Chang B, Yang F, Guo X, Cai KQ, Xiao XS, Wang H, Sen S, Hung MC, Mills GB, Chang S, Multani AS, Mercado-Uribe I and Liu J: Aurora kinase A promotes ovarian tumorigenesis through dysregulation of the cell cycle and suppression of BRCA2. Clin Cancer Res 16: 3171-3181, 2010.
8. Shankar S, Nall D, Tang SN, Meeker D, Passarini J, Sharma J and Srivastava RK: Resveratrol inhibits pancreatic cancer stem cell characteristics in human and KrasG12D transgenic mice by inhibiting pluripotency maintaining factors and epithelialmesenchymal transition. PLoS One 6: e16530, 2011.

9. Fremont L: Biological effects of resveratrol. Life Sci 66: 663-673, 2000.

10. Fukui M, Yamabe N and Zhu BT: Resveratrol attenuates the anticancer efficacy of paclitaxel in human breast cancer cells in vitro and in vivo. Eur J Cancer 46: 1882-1891, 2010.

11. Kopp P: Resveratrol, a phytoestrogen found in red wine. A possible explanation for the conundrum of the 'French paradox'? Eur J Endocrinol 138: 619-620, 1998.

12. Roldan A, Palacios V, Caro I and Perez L: Resveratrol content of Palomino fino grapes: influence of vintage and fungal infection. J Agric Food Chem 51: 1464-1468, 2003.

13. Yi CO, Jeon BT, Shin HJ, Jeong EA, Chang KC, Lee JE, Lee DH, Kim HJ, Kang SS, Cho GJ, Choi WS and Roh GS: Resveratrol activates AMPK and suppresses LPS-induced NF-kappaBdependent COX-2 activation in RAW 264.7 macrophage cells. Anat Cell Biol 44: 194-203, 2011.

14. Kim KY, Yi BR, Lee HR, Kang NH, Jeung EB, Kim SU and Choi KC: Stem cells with fused gene expression of cytosine deaminase and interferon- $\beta$ migrate to human gastric cancer cells and result in synergistic growth inhibition for potential therapeutic use. Int J Oncol 40: 1097-1104, 2011.

15. Yi BR, Kang NH, Hwang KA, Kim SU, Jeung EB and Choi KC: Antitumor therapeutic effects of cytosine deaminase and interferon-beta against endometrial cancer cells using genetically engineered stem cells in vitro. Anticancer Res 31: 2853-2861, 2011.

16. Yi BR, O SN, Kang NH, Hwang KA, Kim SU, Jeung EB, Kim YB, Heo GJ and Choi KC: Genetically engineered stem cells expressing cytosine deaminase and interferon- $\beta$ migrate to human lung cancer cells and have potentially therapeutic antitumor effects. Int J Oncol 39: 833-839, 2011.

17. Kang NH, Yi BR, Lim SY, Hwang KA, Baek YS, Kang KS and Choi KC: Human amniotic membrane-derived epithelial stem cells display anticancer activity in BALB/c female nude mice bearing disseminated breast cancer xenografts. Int J Oncol: Feb, 2012 (E-pub ahead of print).

18. Hwang KA, Hwang YL, Lee MH, Kim NR, Roh SS, Lee Y, Kim CD, Lee JH and Choi KC: Adenosine stimulates growth of dermal papilla and lengthens the anagen phase by increasing the cysteine level via fibroblast growth factors 2 and 7 in an organ culture of mouse vibrissae hair follicles. Int J Mol Med 29: 195-201, 2011.

19. Shanle EK and Xu W: Endocrine disrupting chemicals targeting estrogen receptor signaling: identification and mechanisms of action. Chem Res Toxicol 24: 6-19, 2011.

20. Lee MH, Choi BY, Kundu JK, Shin YK, Na HK and Surh YJ: Resveratrol suppresses growth of human ovarian cancer cells in culture and in a murine xenograft model: eukaryotic elongation factor 1A2 as a potential target. Cancer Res 69: 7449-7458, 2009.

21. Colombo N, Van Gorp T, Parma G, Amant F, Gatta G, Sessa C and Vergote I: Ovarian cancer. Crit Rev Oncol Hematol 60: 159-179, 2006.

22. Roy SK, Chen Q, Fu J, Shankar S and Srivastava RK: Resveratrol inhibits growth of orthotopic pancreatic tumors through activation of FOXO transcription factors. PLoS One 6: e25166, 2011.

23. Vanamala J, Reddivari L, Radhakrishnan S and Tarver C: Resveratrol suppresses IGF-1 induced human colon cancer cell proliferation and elevates apoptosis via suppression of IGF-1R/ Wnt and activation of p53 signaling pathways. BMC Cancer 10: $238,2010$.

24. Liu D, Liu J, Lin B, Liu S, Hou R, Hao Y, Liu Q, Zhang S and Iwamori M: Lewis y regulate cell cycle related factors in ovarian carcinoma cell RMG-I in vitro via ERK and Akt signaling pathways. Int J Mol Sci 13: 828-839, 2012. 